\title{
Predictors of home oxygen duration in chronic neonatal lung disease
}

\author{
Matthew Wong ${ }^{1}$, Melissa Neylan², Gordon Williams ${ }^{1}$, Syeda Zahir $^{2}$, and Jasneek Chawla ${ }^{1}$ \\ ${ }^{1}$ Children's Health Queensland Hospital and Health Service \\ ${ }^{2}$ Queensland Health Centre for Children's Health Research
}

January 4, 2021

\begin{abstract}
Aims: In infants with chronic neonatal lung disease (CNLD), we aimed to identify predictors of home oxygen duration, predictors of discharge oxygen flow rates and the association of oxygen flow rates with respiratory outcomes.

Methods: Retrospective review of infants with CNLD requiring home oxygen in 2016 and 2017. Hazard ratios (HR) were estimated from Cox proportional hazards regression models in the cohort. A multinomial logistic regression model examined the effects of maternal and infant variables on discharge oxygen flow rates. Kruskal-Wallis test with univariate linear regression and Fisher's exact test with binomial univariate logistic regression were used to examine associations between oxygen flow groups and post-discharge clinical variables.

Results: 149 infants were included. Median corrected gestational age (CGA) at oxygen cessation was 6.8 months (IQR 4.4) with $87.2 \%$ of infants weaned by 12 months CGA. Shorter initial neonatal intensive care unit (NICU) stay predicted faster oxygen weaning at 9 months (HR 0.99, 95\% CI 0.98-1.00, $p=0.02$ ) and 12 months (HR 0.99, 95\%CI 0.98-1.00, $p=0.02$ ). Infants with hypercarbia at discharge or discharged from NICU at higher CGA had higher odds of requiring [?]200mL/min relative to [?]125 mL/min oxygen. Infants discharged with $>250 \mathrm{~mL} / \mathrm{min}$ oxygen were more likely to have a respiratory related admission before two years chronologic age.

Conclusion: Shorter initial NICU stay was the best predictor of earlier home oxygen cessation. At NICU discharge, infants with hypercarbia or a higher CGA may require more home oxygen and experience more respiratory related hospital admission in the first two years of chronological age.
\end{abstract}

\section{MAIN TEXT}

\section{Introduction}

Chronic neonatal lung disease (CNLD) is defined clinically as a need for supplemental oxygen therapy or positive pressure support at 36 weeks CGA, at 56 days chronological age if born at [?] 32 weeks gestational age) or at time of hospital discharge. ${ }^{1}$ It is pathologically known as bronchopulmonary dysplasia (BPD), a common respiratory complication of low birth weight preterm infants who experience arrested lung development and are susceptible to lung inflammation secondary to early supplemental oxygen, positive pressure ventilation, and sepsis in the postnatal period. ${ }^{2}$ CNLD and BPD are used interchangeably in the literature with CNLD (or simply chronic lung disease) being the preferred terminology used by the Australian and New Zealand Neonatal Network (ANZNN). ${ }^{3}$

The worldwide incidence of CNLD has been increasing since 1993 due to an increased survival of extreme preterm infants. ${ }^{4}$ The 2017 ANZNN prevalence of CNLD was $30.3 \%$ with 418 (20.8\%) babies with CNLD requiring home oxygen therapy. ${ }^{3}$ Of those requiring home oxygen, $340(81.3 \%)$ also had very low birthweight $(<1,500 \mathrm{~g}) .^{3}$ In Canada and the United States, the annual reported prevalence of extremely premature infants with CNLD and chronic oxygen dependency is between 25 and 59\%.5-8 Home oxygen facilitates earlier hospital discharge and is generally favored as it reduces the risk of nosocomial infections and may 
improve quality of life in the comfort of a home environment. ${ }^{9,10}$ Duration of home oxygen therapy varies widely due to unstandardized weaning practices in CNLD. ${ }^{5}$

Population-based cohort studies have identified predictors of requiring home oxygen at hospital discharge but to our knowledge, no studies have evaluated predictors of home oxygen therapy duration in CNLD. ${ }^{6,7}$ Thus, the primary aim of this study was to identify predictors of home oxygen therapy duration in premature infants with CNLD. Secondary aims were to identify predictors of home oxygen flow rates at time of hospital discharge and to identify if discharge oxygen flow rates were associated with post-discharge respiratory outcomes up to two years chronological age.

\section{Methods}

\section{Study Participants}

This retrospective cohort study identified all infants with a primary diagnosis of moderate or severe CNLD, who required home oxygen therapy, and were managed at the Queensland Children's Hospital (QCH) between January 2016 and December 2017. All infants were managed according to the standard home oxygen protocol within our center (Appendix 1). Demographic and clinical information was obtained from the patient's integrated electronic medical record (iEMR). Post-discharge clinical variables were collected until the infants reached 24 months chronological age. Small for gestational age (SGA) was defined as being less than $10^{\text {th }}$ percentile for birth weight using the Fenton preterm growth chart. ${ }^{11}$ This study was approved by the Children's Health Queensland Hospital and Health Service Human Research Ethics Committee.

\section{Outcomes}

The primary outcome of this study was all-cause home oxygen cessation at three, six, nine, and twelve months corrected gestational age (CGA). Time on oxygen was calculated from birth to CGA at oxygen cessation using CGA in days as a timescale. Infants who did not achieve the outcome at 12 months CGA or those who were lost to follow up before the end of study $(n=20)$ were censored.

A secondary outcome was to assess maternal and infant predictors of home oxygen flow rate required at hospital discharge. Another was to examine how oxygen flow rates at discharge predicted various post-discharge variables up to 24 months chronological age which included respiratory related emergency department (ED) presentations, respiratory related hospital admissions, doctor documented wheeze, prescribed oral corticosteroids, prescribed inhaled corticosteroids, pediatric intensive care unit (PICU) admissions, and pulmonary hypertension.

\section{Statistical Analyses}

The study population characteristics were described by summary statistics (Table 1). For continuous variables, measures of central tendency (mean/median) and spread (standard deviation/interquartile range) were presented. Mean/standard deviation (SD) were presented for normally distributed continuous variables and median/interquartile range (IQR) for non-normally distributed variables. Normality was checked by conducting the Shapiro-Wilks test and visual inspection of histograms. Frequencies and proportions were presented for categorical variables.

Cox proportional hazards regression models were used to estimate effects of each a priori predictor of home oxygen duration on home oxygen cessation, with simultaneous adjustment for potential confounders in multivariable models. Hazard ratios (HR) with $95 \%$ confidence intervals (CI) were calculated. Four Cox-models were built for four different time on oxygen cut-off points: from baseline to three, six, nine, 
and twelve months CGA respectively. The proportional hazards assumption was assessed by examining the relationship between the scaled Schoenfeld residuals and time on oxygen and the overall fit of the models was tested by examining the Cox-Snell residuals and evidence of non-proportional hazards for oxygen flow was found. Hence, the baseline hazard function was stratified by oxygen flow group and proportional hazards assumption was satisfied by fitting a stratified Cox-regression model. Covariates included for adjustment in multivariable models were GA, birth weight, partial pressure of carbon dioxide $\left(\mathrm{pCO}_{2}\right)$ at term CGA, invasive ventilation total respiratory support and length of initial NICU hospital stay in days.

We assessed the potential effects of different maternal and infant variables on oxygen flow rates at hospital discharge using multinomial logistic regression. Patients were grouped into three oxygen flow groups based on their oxygen flow requirements at discharge: a) [?] $125 \mathrm{~mL} / \mathrm{min}$; b) $126 \mathrm{~mL} / \mathrm{min}$ to $250 \mathrm{~mL} / \mathrm{min}$; c) 251 $\mathrm{mL} / \mathrm{min}$ to $750 \mathrm{~mL} / \mathrm{min}$. Basic inferential statistical tests were conducted to select variables with significant differences between the three oxygen flow groups in the final multinomial logistic regression model. A Kruskal-Wallis test was used for continuous variables (i.e., birth weight in grams, pCO2 at term CGA in $\mathrm{mmHg}$, total duration of respiratory support in days, length of hospital stay in days and CGA at hospital discharge in weeks). A Chi-Square test was used for categorical variables including GA [?] 28 weeks and maternal PROM. Odds ratios (OR) with $95 \%$ CI were calculated and presented for the final multinomial logistic model. Modelled estimates of the sensitivity, specificity, positive predictive value (PPV) and negative predictive value (NPV) predictive values were derived and presented as Appendix 2.

The Kruskal-Wallis test was used to examine the association between the three oxygen flow groups and continuous post-discharge variables. A univariate linear regression was then conducted to identify the effect of oxygen flow and continuous post-discharge variables. For oxygen flow groups and categorical post-discharge variables, a Fisher's Exact test and a binomial univariate logistic regression were used

\section{Results}

A total of 149 infants (63.1\% males) were included in the analyses (Table 1). In our cohort, infants with CNLD requiring home oxygen had a median GA at birth of 26 weeks (IQR 3) and BW 779 grams (IQR 331) with $13(8.7 \%)$ born SGA. The median length of invasive ventilation during the neonatal period was 17.9 days (IQR 29.4) and mean length of total respiratory support was 73.3 days (SD 28.6) with $53 \%$ requiring high frequency oscillation ventilation. At term CGA, the mean $\mathrm{pCO}_{2}$ was $53.9 \mathrm{mmHg}$ (SD 7.1). At discharge, the median CGA was 42 weeks (IQR 4.5) with $70.5 \%$ of infants requiring $250 \mathrm{~mL} / \mathrm{min}$ of oxygen flow or less at discharge. The median CGA of oxygen weaning was 6.8 months CGA (IQR 4.4) with 87.2\% of infants able to wean off oxygen by 12 months CGA.

Cox proportional hazards regression models for clinical predictors of oxygen weaning at three, six, nine and twelve months CGA with a priori variables of $\mathrm{GA}, \mathrm{BW}, \mathrm{pCO}_{2}$ at term CGA, duration of invasive ventilation, total duration of respiratory support, length of hospital stay and oxygen flow group at discharge are shown in Table 2. The models indicated that shorter length of hospital stay was a statistically significant predictor of faster oxygen weaning at CGA 9 months (HR 0.99, 95\% CI $0.98-1.00, p=0.02$ ) and at 12 months (HR 0.99, 95\% CI $0.98-1.00, p=0.02$ ) (Table 2). Length of hospital stay was not found to be a significant predictor of oxygen weaning at CGA 3 months (HR 1.01, 95\% CI $0.97-1.05, p=0.65$ ) or 6 months (HR $0.99,95 \%$ CI 0.97-1.00, $p=0.16$ ). Higher GA, higher $\mathrm{BW}$, lower $\mathrm{pCO}_{2}$ at term CGA, fewer invasive ventilation days and fewer total days on respiratory support were not significant predictors of faster oxygen weaning at any time point (Table 2).

Our multinomial logistic regression (Table 3) indicated that infants has higher odds of being discharged with 
200-250 mL/min (OR 1.099, 95\% CI 1.02-1.19, $p=0.02$ ) or $251-750 \mathrm{~mL} / \mathrm{min}$ (OR 1.13, 95\% CI 1.04-1.23, $p=0.004$ ) home oxygen relative to [?] $125 \mathrm{~mL} / \mathrm{min}$ with increasing measured pCO2 at term CGA when all other variables were held constant. With each week increase in CGA at hospital discharge, there were higher odds of being discharged on $200-250 \mathrm{~mL} / \mathrm{min}$ (OR 1.34, 95\% CI 1.09-1.63, $p=0.005$ ) or 251-750 $\mathrm{mL} / \mathrm{min}$ (OR 1.51, 95\% CI 1.21-1.88, $p<0.001$ ) relative to [?] $125 \mathrm{~mL} / \mathrm{min}$ when all other variables were held constant. With every day increase in length of hospital stay, infants had lower odds of being discharged on $200-250 \mathrm{~mL} / \mathrm{min}($ OR $0.95,95 \%$ CI $0.91-0.99, p=0.02)$ or $251-750 \mathrm{~mL} / \mathrm{min}$ (OR 0.94, 95\% CI 0.90-0.99, $p=0.01$ ) relative to [?] $125 \mathrm{~mL} / \mathrm{min}$ when all other variables were held constant. Our results also suggest that infants born to mothers with PROM had lower odds of being discharged on $200-250 \mathrm{~mL} / \mathrm{min}$ home oxygen (OR 0.36, 95\% CI 0.14-0.91, $p=0.032$ ) compared to mothers without PROM when all other variables were held constant (Table 3).

For our model, accuracy was around 55\% (CI 46-63\%) with performance of the model using diagnostic measures outline in Appendix 2. Sample sizes were low in some groups which is reflected by the accuracy of the model.

We found significant associations of oxygen flow rate at discharge with respiratory related hospital admissions $(p<0.01)$ and PICU admissions $(p=0.05)$ up to two years of chronological age (Table 4$)$. We estimated that infants discharged on 251-750 mL/min of home oxygen had 6.08 times higher odds (95\% CI, 2.20-18.48, $p<0.001$ ) of being admitted (at least once) with a respiratory related illness within the first two years of chronological age (Appendix 3).

\section{Discussion}

Many of the variables that predict home oxygen in CNLD such as early gestational age, low birth weight, invasive ventilation time or total duration of respiratory support did not predict oxygen weaning within 12 months CGA. ${ }^{6,7}$ Our finding that infants who were discharged on higher oxygen flow rates required home oxygen therapy for longer is similar to what has been reported previously. ${ }^{12}$ Variables typically associated with severity of CNLD like high frequency oscillation ventilation, early gestational age, or hypercapnia at term CGA did not correlate with the duration of home oxygen therapy. Therefore, infants should not be presumed to need longer oxygen therapy based on ventilation requirements in NICU.

In our cohort, prolonged neonatal hospital stay was associated with increased duration of home oxygen therapy. The reported duration of home oxygen therapy in CNLD worldwide over the last 40 years is between 2 and 13.5 months (Table 5). ${ }^{12-24}$ When home oxygen was initially introduced, weaning was based on a target partial pressure of arterial oxyhemoglobin $\left(\mathrm{PaO}_{2}\right)$ of $55-60 \mathrm{mmHg} .{ }^{13-15}$ Continuous overnight pulse oximetry has become more commonly used to guide oxygen weaning with expert consensus suggesting targeting a mean peripheral arterial hemoglobin saturation $\left(\mathrm{SpO}_{2}\right)>93-95 \%$ and [?] 5\% of the overnight oximetry time spent with $\mathrm{SpO}_{2}$ [?] $90 \%$ or $\mathrm{SpO}_{2}$ [?] 93\%. ${ }^{25-28}$ However, there is a significant lack of evidence supporting these recommendations and thus home oxygen weaning in CNLD remains highly variable between health services. ${ }^{29,30}$

Several service provision variables likely contribute duration of oxygen therapy for these infants. This includes who oversees oxygen weaning following discharge from neonatal care and the frequency of follow up visits in the first year of life. Models of care differ across centers with neonatology, general pediatrics, general practitioners, respiratory, and sleep specialists all involved in patient care to varying degrees. Some centers may even have oxygen weaning unsupervised by a medical provider. ${ }^{12,30}$ At our center, infants are reviewed every 6-8 weeks post-discharge (Appendix 1) and the median age of home oxygen cessation was 6.8 
months CGA (IQR 4.4), with $51 \%$ of infants discharged on oxygen flow rates of 200-250 mL/min (Table 1). In contrast, centers where a clinical nurse undertakes weaning in the community may wean oxygen slower and be affected by the follow up frequency.

Centers that rely on polysomnography (PSG) assessment for oxygen weaning report prolonged duration of home oxygen therapy possibly due to PSG waiting times (Table 5). ${ }^{12,31}$ In our experience, the majority of infants with CNLD can be safely weaned using overnight oximetry monitoring and PSG is reserved for infants requiring more than $500 \mathrm{~mL} / \mathrm{min}$ of supplemental oxygen at time of discharge, infants with a clinical suspicion of comorbid obstructive sleep apnea (OSA), or when there is concern that an additional etiology contributes to hypoxemia.

The current lack of standardization of home oxygen initiation and weaning makes it difficult to assess outcomes in these infants. Currently, no evidence exists to suggest that one method of weaning supplemental oxygen therapy is superior to another and to our knowledge no studies have been able to evaluate long-term outcomes following a standardized weaning protocol. This highlights the need for prospective longitudinal studies in view of the continued increase in the number of preterm infants requiring home oxygen. ${ }^{32}$

Infants with CNLD are known to be at higher risk of lower respiratory tract infections (especially respiratory syncytial virus) and those receiving home oxygen therapy are $10 \%$ more likely to be readmitted for a respiratory illness with $49-58 \%$ of infants with severe CNLD requiring readmission within the first two years of life. ${ }^{5,33,34}$ In our study, infants discharged on higher oxygen flow levels required more admissions (including PICU) which is in keeping with findings in other studies. ${ }^{5}$ It is important for clinicians to monitor these higher risk infants closely during this early period and ensuring families are aware of the increased vulnerability if an infant is discharged on [?] $500 \mathrm{~mL} / \mathrm{min}$ of oxygen. The number of ED visits within the first two years did not correlate with discharge oxygen flow rates, perhaps indicating that home oxygen may help reduce severity of subsequent respiratory illnesses.

Wheeze and bronchial hyper-responsivity are well described following preterm birth ${ }^{35-38}$ and infants with CNLD are more likely to receive additional oxygen, bronchodilators and inhaled steroids within the first year of life. ${ }^{23}$ In our study, doctor documented wheeze and corticosteroid use (inhaled or oral) in the first 2 years of life were not significantly associated with duration of oxygen therapy or oxygen flow rates at discharge. Further longitudinal follow up of our cohort would be required to determine if such presentations occur later in genetically predisposed individuals, with prematurity and CNLD increasing risk during pre-school years. All infants with CNLD requiring home oxygen at our center receive respiratory syncytial virus immunoglobulin prophylaxis during the winter months which may have reduced wheezy hospital presentations compared to other studies.

Infants were more likely to be discharge on higher levels of oxygen therapy ([?] $200 \mathrm{~mL} / \mathrm{min}$ ) if they had higher pCO2 measured at term CGA and increased CGA at hospital discharge. In our cohort, 30.9\% ( $=46)$ had moderate hypercapnia $\left(\mathrm{pCO}_{2} 55-65 \mathrm{mmHg}\right)$ and $6 \%(\mathrm{n}=9)$ had severe hypercapnia $\left(\mathrm{pCO}_{2}>65 \mathrm{mmHg}\right)$ measured at term CGA (Table 1). Moderate to severe hypercapnia was not predictive of home oxygen cessation but was significantly associated with requiring $>250 \mathrm{~mL} / \mathrm{min}$ oxygen at discharge (Table 3 ). In a sub-analysis, infants with $\mathrm{pCO}_{2}$ [?] $55 \mathrm{mmHg}$ had a median time to oxygen weaning of 6.3 months $(95 \%$ CI, 5.7-7.0) whereas patients with $\mathrm{pCO}_{2}>55 \mathrm{mmHg}$ had a median time of 8.4 months (95\% CI, 7.7-9.1) (Appendix 4). The differences were statistically significant at our time on oxygen cut-off points of CGA 6 months ( $p=0.001), 9$ months $(p=0.035)$, and 12 months $(p=0.034)$. This information may be useful clinically 
to guide parent's expectations of a longer duration of oxygen therapy in those who have hypercarbia at term CGA.

It is unclear whether alternative or additional respiratory support would be beneficial in infants with hypercarbia as there are no studies assessing the impact of hypercarbia and level of respiratory support on outcomes in CNLD. Centers may consider PSG to evaluate the need for pressure support in infants who have evidence of alveolar hypoventilation (more than $25 \%$ of the total sleep time with $\left.\mathrm{pCO}_{2}>50 \mathrm{mmHg}\right)^{41}$ but it is unclear which infants with moderate to severe hypercapnia benefit from pressure support over supplemental oxygen. A Canadian retrospective cohort study identified that of infants born at GA $<29$ weeks, only $0.9 \%$ and $0.6 \%$ were discharged on non-invasive ventilation (NIV) or tracheostomy ventilation respectively. ${ }^{42}$ The indications for initiating NIV were not stated. After laryngomalacia, CNLD was the second most common indication for home CPAP over a period of 10 years at Westmead Children's Hospital. ${ }^{43}$ No specific gas exchange or PSG criteria for initiating CPAP was mentioned. Future work to explore practices for NIV therapy in infants with CNLD across centers would be a first step in determining whether this is an option that should be offered more readily in this population and whether this impacts longer term outcomes.

Our study is limited by its retrospective nature but is strengthened by the inclusion of a reasonable sample size. The multinomial logistic regression model to predict oxygen flow rates at discharge would need to be validated on a larger cohort of patients as the lower accuracy was affected by the smaller sample size in some comparative groups. There is selection bias as only infants managed at a single center were included for analysis. We recognize that length of oxygen therapy and weaning practices are often based on the anecdotal experience of individual clinicians due to the lack of evidence in this area. This is consistent with practice in other centers as there are currently no standardized protocols to guide weaning of home oxygen therapy in infants with CNLD. Recall bias is minimized by our multidisciplinary oxygen clinic following a consistent follow up and weaning process (Appendix 1). Although our study only describes associations between a priori maternal and infant variable with home oxygen duration and oxygen flow rates, it provides insightful information about which infants may require prolonged oxygen therapy or experience more hospital readmissions.

\section{Conclusions}

This study provides information which may be of use for clinicians when advising families of infants with CNLD being discharged on home supplemental oxygen therapy. Shorter length of initial NICU stay appears to predict shorter duration of home oxygen therapy and was associated with lower odds of needing [?] 200 $\mathrm{mL} / \mathrm{min}$ at discharge. Infants with higher CGA at discharge or hypercarbia were more likely to need higher levels of home oxygen therapy. Close monitoring is recommended for infants discharged on higher flows of oxygen therapy (e.g. $>250 \mathrm{~mL} / \mathrm{min}$ ) as these infants have a higher risk of respiratory related hospital and PICU admissions within the first two years. Additionally, our study highlights the variability in practice and need for standardization of oxygen weaning process for infants with CNLD in order to improve outcomes for this increasing population.

\section{References}

1. Jobe AH, Bancalari E. Bronchopulmonary dysplasia. Am J Respir Crit Care Med. 2001;163(7):17231729.

2. Thebaud B, Goss KN, Laughon M, et al. Bronchopulmonary dysplasia. Nat Rev Dis Primers. $2019 ; 5(1): 78$.

3. Chow SSWC, P.; Chambers, G.M.; Lui, K. Report of the Australian and New Zealand Neonatal Network 2017. Sydney: UNSW;2019. 
4. Stoll BJ, Hansen NI, Bell EF, et al. Trends in Care Practices, Morbidity, and Mortality of Extremely Preterm Neonates, 1993-2012. JAMA. 2015;314(10):1039-1051.

5. DeMauro SB, Jensen EA, Bann CM, et al. Home Oxygen and 2-Year Outcomes of Preterm Infants With Bronchopulmonary Dysplasia. Pediatrics. 2019;143(5).

6. Ejiawoko A, Lee HC, Lu T, Lagatta J. Home Oxygen Use for Preterm Infants with Bronchopulmonary Dysplasia in California. J Pediatr. 2019;210:55-62 e51.

7. Lagatta J, Clark R, Spitzer A. Clinical predictors and institutional variation in home oxygen use in preterm infants. J Pediatr. 2012;160(2):232-238.

8. Lodha A, Sauve R, Bhandari V, et al. Need for supplemental oxygen at discharge in infants with bronchopulmonary dysplasia is not associated with worse neurodevelopmental outcomes at 3 years corrected age. PLoS One. 2014;9(3):e90843.

9. Lau R, Crump RT, Brousseau DC, et al. Parent Preferences Regarding Home Oxygen Use for Infants with Bronchopulmonary Dysplasia. J Pediatr. 2019;213:30-37 e33.

10. Balfour-Lynn IM, Primhak RA, Shaw BN. Home oxygen for children: who, how and when? Thorax. 2005;60(1):76-81.

11. Fenton TR, Kim JH. A systematic review and meta-analysis to revise the Fenton growth chart for preterm infants. BMC Pediatr. 2013;13:59.

12. Yeh J, McGrath-Morrow SA, Collaco JM. Oxygen weaning after hospital discharge in children with bronchopulmonary dysplasia. Pediatr Pulmonol. 2016;51(11):1206-1211.

13. Pinney MA, Cotton EK. Home management of bronchopulmonary dysplasia. Pediatrics. 1976;58(6):856-859.

14. Campbell AN, Zarfin Y, Groenveld M, Bryan MH. Low flow oxygen therapy in infants. Arch Dis Child. 1983;58(10):795-798.

15. Abman SH, Accurso FJ, Koops BL. Experience with home oxygen in the management of infants with bronchopulmonary dysplasia. Clin Pediatr (Phila). 1984;23(9):471-476.

16. Hudak BB, Allen MC, Hudak ML, Loughlin GM. Home oxygen therapy for chronic lung disease in extremely low-birth-weight infants. Am J Dis Child. 1989;143(3):357-360.

17. Sauve RS, McMillan DD, Mitchell I, Creighton D, Hindle NW, Young L. Home oxygen therapy. Outcome of infants discharged from NICU on continuous treatment. Clin Pediatr (Phila). 1989;28(3):113118.

18. Silva DT, Hagan R, Sly PD. Home oxygen management of neonatal chronic lung disease in Western Australia. J Paediatr Child Health. 1995;31(3):185-188.

19. Baraldi E, Carra S, Vencato F, et al. Home oxygen therapy in infants with bronchopulmonary dysplasia: a prospective study. Eur J Pediatr. 1997;156(11):878-882.

20. Bertrand P, Alvarez C, Fabres J, Simonetti M, Sanchez I. [Home oxygen therapy in children with chronic respiratory failure]. Rev Med Chil. 1998;126(3):284-292.

21. Norzila MZ, Azizi BH, Norrashidah AW, Yeoh NM, Deng CT. Home oxygen therapy for children with chronic lung diseases. Med J Malaysia. 2001;56(2):151-157.

22. Saletti A, Stick S, Doherty D, Simmer K. Home oxygen therapy after preterm birth in Western Australia. J Paediatr Child Health. 2004;40(9-10):519-523.

23. Hennessy EM, Bracewell MA, Wood N, et al. Respiratory health in pre-school and school age children following extremely preterm birth. Arch Dis Child. 2008;93(12):1037-1043. 
24. Batey N, Batra D, Dorling J, Bhatt JM. Impact of a protocol-driven unified service for neonates with bronchopulmonary dysplasia. ERJ Open Res. 2019;5(1).

25. Kapur N, Nixon G, Robinson P, et al. Respiratory management of infants with chronic neonatal lung disease beyond the NICU: A position statement from the Thoracic Society of Australia and New Zealand. Respirology. 2020.

26. Balfour-Lynn IM, Field DJ, Gringras P, et al. BTS guidelines for home oxygen in children. Thorax. 2009;64 Suppl 2:ii1-26.

27. Hayes D, Jr., Wilson KC, Krivchenia K, et al. Home Oxygen Therapy for Children. An Official American Thoracic Society Clinical Practice Guideline. Am J Respir Crit Care Med. 2019;199(3):e5-e23.

28. Thoracic Society of A, New Z, Fitzgerald DA, et al. Infants with chronic neonatal lung disease: recommendations for the use of home oxygen therapy. Med J Aust. 2008;189(10):578-582.

29. Wong MD, Chung H, Chawla J. Using continuous overnight pulse oximetry to guide home oxygen therapy in chronic neonatal lung disease. J Paediatr Child Health. 2020;56(2):309-316.

30. Palm K, Simoneau T, Sawicki G, Rhein L. Assessment of current strategies for weaning premature infants from supplemental oxygen in the outpatient setting. Adv Neonatal Care. 2011;11(5):349-356.

31. Kulkarni G, de Waal K, Grahame S, et al. Polysomnography for the management of oxygen supplementation therapy in infants with chronic lung disease of prematurity. J Matern Fetal Neonatal Med. 2018:1-7.

32. Hayes D, Jr., Wilson KC, Balfour-Lynn IM. Need for international standardization of home oxygen therapy in children. Pediatr Pulmonol. 2019.

33. Abman SH, Collaco JM, Shepherd EG, et al. Interdisciplinary Care of Children with Severe Bronchopulmonary Dysplasia. J Pediatr. 2017;181:12-28 e11.

34. Carbonell-Estrany X, Quero J, Bustos G, et al. Rehospitalization because of respiratory syncytial virus infection in premature infants younger than 33 weeks of gestation: a prospective study. IRIS Study Group. Pediatr Infect Dis J. 2000;19(7):592-597.

35. Castro-Rodriguez JA, Forno E, Rodriguez-Martinez CE, Celedon JC. Risk and Protective Factors for Childhood Asthma: What Is the Evidence? J Allergy Clin Immunol Pract. 2016;4(6):1111-1122.

36. Leps C, Carson C, Quigley MA. Gestational age at birth and wheezing trajectories at 3-11 years. Arch Dis Child. 2018;103(12):1138-1144.

37. Perez-Yarza EG, Moreno-Galdo A, Ramilo O, et al. Risk factors for bronchiolitis, recurrent wheezing, and related hospitalization in preterm infants during the first year of life. Pediatr Allergy Immunol. 2015;26(8):797-804.

38. Been JV, Lugtenberg MJ, Smets E, et al. Preterm birth and childhood wheezing disorders: a systematic review and meta-analysis. PLoS Med. 2014;11(1):e1001596.

39. Berkelhamer SK, Mestan KK, Steinhorn R. An update on the diagnosis and management of bronchopulmonary dysplasia (BPD)-associated pulmonary hypertension. Semin Perinatol. 2018;42(7):432443.

40. Al-Ghanem G, Shah P, Thomas S, et al. Bronchopulmonary dysplasia and pulmonary hypertension: a meta-analysis. J Perinatol. 2017;37(4):414-419.

41. Berry RQ, SF; Abreu, AR; et al for the American Academy of Sleep Medicine. The AASM Manual for the Scoring of Sleep and Associated Events: Rules, Terminology and Technical Specifications. Version 2.6. In: Darien, IL: American Academy of Sleep Medicine; 2020. 
42. Nassel D, Chartrand C, Dore-Bergeron MJ, et al. Very Preterm Infants with Technological Dependence at Home: Impact on Resource Use and Family. Neonatology. 2019;115(4):363-370.

43. Al-Iede M, Kumaran R, Waters K. Home continuous positive airway pressure for cardiopulmonary indications in infants and children. Sleep Med. 2018;48:86-92.

\section{Hosted file}

Home-02-CNLD-PPUL-table1-R2-25Dec2020.pdf available at https://authorea.com/users/360743/ articles/482285-predictors-of-home-oxygen-duration-in-chronic-neonatal-lung-disease

\section{Hosted file}

Home-02-CNLD-PPUL-table2-R2-25Dec2020.pdf available at https://authorea.com/users/360743/ articles/482285-predictors-of-home-oxygen-duration-in-chronic-neonatal-lung-disease

\section{Hosted file}

Home-02-CNLD-PPUL-table3-R2-25Dec2020.pdf available at https://authorea.com/users/360743/ articles/482285-predictors-of-home-oxygen-duration-in-chronic-neonatal-lung-disease

\section{Hosted file}

Home-02-CNLD-PPUL-table4-R2-25Dec2020.pdf available at https://authorea.com/users/360743/ articles/482285-predictors-of-home-oxygen-duration-in-chronic-neonatal-lung-disease

\section{Hosted file}

Home-02-CNLD-PPUL-table5-R2-25Dec2020.pdf available at https://authorea.com/users/360743/ articles/482285-predictors-of-home-oxygen-duration-in-chronic-neonatal-lung-disease 